\title{
La professionnalisation du rugby français. Pouvoir économique et lien social
}

Jean-Bernard Marie Moles

\section{(2) OpenEdition}

1 Journals

Édition électronique

URL : http://journals.openedition.org/corpsetculture/470

DOI : 10.4000/corpsetculture.470

ISSN : $1777-5337$

Éditeur

Association Corps et Culture

Édition imprimée

Date de publication : 1 juin 1998

ISSN : 1268-5631

Référence électronique

Jean-Bernard Marie Moles, "La professionnalisation du rugby français. Pouvoir économique et lien social », Corps et culture [En ligne], Numéro 3| 1998, mis en ligne le 24 septembre 2007, consulté le 07 septembre 2020. URL : http://journals.openedition.org/corpsetculture/470 ; DOI : https://doi.org/ $10.4000 /$ corpsetculture.470

Ce document a été généré automatiquement le 7 septembre 2020

(c) tous droits réservés 


\title{
La professionnalisation du rugby français. Pouvoir économique et lien social
}

\author{
Jean-Bernard Marie Moles
}

Introduction: le rugby français aspiré dans le tourbillon novateur venu de l'hémisphère sud

1 C'est en 1987 que se déroule aux Antipodes, en Nouvelle-Zélande et en Australie, la première Coupe du Monde de Rugby à XV, suivie en 1991 par celle mise en place par l'hémisphère Nord dont la Grande-Bretagne et la France. L'International Board, institution patriarcale regroupant Anglais, Ecossais, Gallois, Irlandais et Français, légifère le football-rugby depuis sa création en 1890 et son veto sur le «manque à gagner ", sur un mode fonctionnaliste avec le maintien intangible de son équilibre interne (Pareto V, 1916), la perpétuation de son système de domination (Bourdieu $P$, 1979) excluant jusqu'à nos jours toute cooptation de pays émergents comme l'Italie (qui disputera cependant le Tournoi des VI nations en 2000), l'Argentine, le Canada. L'amateurisme est le seing de l'ordre socio-rugbystique tant dans l'hémisphère Nord que Sud. Ce schème structuralo-fonctionnaliste avec un système homéostatique, soumis à des valeurs (dont l'amateurisme) incarnées dans une culture globale et irrécusable (Parsons T, 1951) permettra de faire du rugby un sport épique, d'équipe, éminemment attrayant, populaire et instructif - le rugby sera une discipline scolaire longtemps obligatoire dans l'ensemble des pays anglo-saxons et fera de l'universitaire sa vitrine d'école de vie - «le rugby, un sport de voyous pratiqué par des gentlemen ». C'est dans cet espace rugbystique que se développeront des liens actifs fondés sur l'acceptation des règles de conduite établies (pas de Coupe du Monde jusqu'en 1987, pas de rugby aux Jeux Olympiques, immutabilité du sacro-saint amateurisme, pas de "squads", de rassemblements ou mise au vert, pas de transfert du XIII au XV) et surtout un fort sentiment d'appartenance à ces mêmes liens qui alors diabolisent l'argent et tissent l'esprit collectif dont se prévalent aujourd'hui, même hypocritement, tous les membres de la famille ovalienne. 
2 Malgré l'universalité vacillante du rugby, le succès médiatique de la seconde Coupe du Monde, effarant, en particulier dans les pays riches, puisque l'une des originalités de ce sport est d'être surtout pratiqué assidûment par des pays membres du G7 plutôt que du Tiers Monde, interpelle le Magnat de la Télévision Anglo-Saxonne, Rupert Murdoch. Qui après quelques tentatives désordonnées de professionnaliser le rugby des Antipodes par divers groupements d'intérêts, propose pour 550 millions de dollars, soit 320 millions de francs par an de droits télés exclusifs et symbiotiques aux Fédérations Australiennes, Néo-Zélandaises et Sud-Africaines d'organiser, essentiellement pour ses chaînes codées, ses propres compétitions, le TRI-SERIES, tournoi triangulaire par matches aller/retour et le TOP 12, tournoi rassemblant des provinces de ces 3 nations. Ce sont les premières compétitions privées de l'histoire du rugby mondial, "without the Board" pour les anglo-saxons. La surabondance du calendrier international n'effraie pas les meilleurs joueurs des 3 nations ayant remporté les trois titres de Champions du Monde (Nouvelle-Zélande en 1987, Australie en 1991, Afrique du Sud en 1995), d'autant moins qu'ils sont largement défrayés par leurs Fédérations de leurs efforts et bonne volonté, $700.000 \mathrm{fr}$. par saison (Escot R., 1996). Le seuil du professionnalisme est franchi.

3 Le Board, conscient de ce processus récursif inaltérable, télé, argent, professionnalisme, auquel les Européens, en particulier les Anglais et les Français prétendent à leur tour, effacera sans tapage, le 14 août 1995 à Paris, définitivement de ses statuts, toute référence alors obligatoire à l'amateurisme se faisant le fourrier d'un séisme socio-rugbystique. Aspirée dans ce tourbillon novateur d'émergence du professionnalisme, l'organisation rugbystique hexagonale, d'inspiration déterministe et holiste va se trouver, dès 1996, ébranlée ex-abrupto par le pôle de l'élite dont la conception isomorphe avec le rugby professionnel de l'hémisphère Sud et le rugby Anglais tend à un dualisme, rugby professionnel et marchand d'un côté, rugby amateur et non-marchand de l'autre. Les liens intra-organisationnels se distendent rapidement et dans le second semestre 1996, ce processus divergent déclenche une multiplication de créations d'institutions informelles puis formelles, contestataires ou alliées de la Fédération et de facto une entropie récurrente, les liens externes qui touchent à l'environnement social, culturel, économique, suivant cette dérégulation extrême au point d'effleurer rapidement le seuil de tolérance maximal.

Car l'arrivée du professionnalisme dans l'hémisphère Nord se déroule dans un contexte d'inadaptation de l'ordre sportif à la mutation du système. Les règles sont constamment modifiées pour privilégier, dans un souci de séduction des télévisions, l'attaque donc le spectacle. La technique des joueurs de haut niveau et leurs qualités physiques sont transcendées par des entraînements quotidiens, la culture du rugby orthodoxe, totalement conforme aux principes et préceptes de la Doctrine du Board et de la Fédération, est elle-même bouleversée dans sa plus sincère authenticité avec des mi-temps qui mutent de 5 à 10 minutes pour allonger les tunnels publicitaires des télévisions. Le championnat de France est mis en jachère durant 3 mois pour laisser place aux Coupes d'Europe et débute en plein été! Le mémorable Challenge Yves-duManoir est banni, suppléé par une Coupe de France seulement réservée aux clubs de 1 ère division (contrairement aux autres disciplines collectives qui englobent l'ensemble de leurs clubs). Deux rencontres (dont Galles/France) du Tournoi des V Nations, représentation symbolique et mythique du rugby de l'hémisphère Nord, sont programmées le dimanche après-midi, Murdoch qui a acheté les droits du XV 
d'Angleterre, récusant le doublement des matches le samedi. En France le rugby devient en 1996 un métier pour 600 joueurs qui signent un contrat professionnel avec leurs clubs. Ils seront 700 pour la saison 97/98 à franchir le seuil de finalité de l'amateurisme : $6.000 \mathrm{Frs}$, la moyenne d'un joueur sous contrat pour cette saison est légèrement supérieur au SMIG, le plus élevé étant supérieur à 70.000 francs mensuels (Midi Olympique et l'Equipe, Décembre 1997).

Cette mutation inéluctable met en porte-à-faux la singularité du rugby qui était de cultiver une exception culturelle indissociablement liée aux éléments de la vie locale, comme la chasse, la viticulture, la bonne chère, la tauromachie. Le rugby est un mode de vie plus qu'un sport basique et disciplinaire. La masse réprouve les dérives du nouveau système ancré sur l'argent (le club d'Argelès-sur-Mer en 1996 dut refuser la montée en 1 ère division Groupe A faute de pouvoir boucler un budget d'1,5 MF) et reconnaît que ce rugby affairiste n'est pas le sien. Mais cette même masse, victime de sa contradiction, de son aversion du mercantilisme et de son rejet de l'élite et du professionnalisme tout en lui demandant son aide et son soutien, va déclencher dans cette ontogenèse, des phénomènes adaptatifs et axiologiques, relayés par la Fédération Française de Rugby, mais évidemment conflictuels avec ceux répandus par l'Elite. La solidarité, nourrie de liens de dépendance mutuelle, implose et ne permet plus de maintenir l'ordre social (Durkheim E., 1937), créant un désordre récurrent et de nouveaux rapports entre les acteurs internes, dirigeants fédéraux, présidents de clubs, entraîneurs, joueurs, supporters et de nouvelles transactions avec les acteurs externes, dirigeants des télévisions, sponsors, équipementiers, public et supporters.

6 Partisans et opposants du rugby professionnel et d'une élite restreinte sont alors immergés dans une foule d'incertitudes, de turbulences qui ébranlent l'organisation fédérale. Le désordre entretenu par des jeux conflictuels, entre néophiles et misonéistes, a du mal à donner naissance à une nouvelle structure organisationnelle faute de stratégies coordinatrices et coopératives. Cette sédimentation organisationnelle du désordre, latente depuis 1996 perdure toujours, le temps de la rémanence et ses effets du professionnalisme venus de l'hémisphère Sud persistant toujours faute d'entente entre les différents et nouveaux acteurs qui habitent le Paradigme Rugbystique.

Quelle nouvelle culture pour le rugby français?

7 C'est pour ces raisons (avec une variable causale ouverte au système, l'alignement obligé du rugby français sur le professionnalisme international, dont celui des Antipodes pour rester compétitif dans les compétitions mondiales, et une variable d'effet, le désordre et la crise qui caractérisent actuellement le système français, bouleversent l'organisation du Rugby Hexagonal et provoquent la nécessité d'une nouvelle structure organisationnelle), que l'espace social se transforme en un bouillonnement de résistances collaboratrices, les partisans d'un jour devenant les opposants du lendemain. L'imperfection des interrelations et rétroactions entre les deux pôles, professionnel et amateur, rationalise cependant la complexité, dont les éléments sont associés mais visent pour l'un, les partisans de l'élite, à la dissociation (Morin E., 1990), pour l'autre, le rugby de masse, à l'union. Même si cette dissociation doit ébranler irréductiblement la cohésion socio-rugbystique et briser les liens sociaux si bien ancrés dans le Peuple d'Ovalie, des mains courantes plutôt que des loges climatisées, avec d'un côté ceux qui courent pour des idées, rugby pro, rugby spectacle, rugby télégénique, rugby d'élite et les autres qui courent pour un idéal, le rugby plaisir, 
le rugby éducatif, le rugby formateur, emblématique, école de la vie, rugby unitaire. D'un côté 1740 clubs, ceux qui composent la masse imposante du rugby unitaire, qui n'entendent rien céder du patrimoine culturel, formateur, festif et qui plutôt que de rompre en appellent à la coopération, de l'autre 40 clubs (ceux des groupes $\mathrm{Al}$ et A2), précurseurs de l'élitisme, de confrontations nouvelles, européennes, planétaires, contingents à une daraise socio-économique de la masse au haut niveau et qui répondent à l'écho par l'émancipation.

Cette approche actionnaliste, avec des stratégies conflictuelles qui opposent deux classes socio-rugbystiques et leurs chefferies, l'élite qualifiée de nantie, monopolisatrice de la richesse, et les « autres » qui en appellent toujours à la solidarité, conduit au-delà de la désintégration du système actuel à l'avènement d'une nouvelle culture, économique, à de nouvelles valeurs et liens sociaux éprouvant de nouvelles différences, apprentissages, rapports. C'est la cristallisation de cette bipolarité, conséquence $\mathrm{du}$ recodage des préceptes $\mathrm{du}$ Board par l'émergence $\mathrm{du}$ professionnalisme, qui nous a guidé dans notre recherche épistémologique à la compréhension de ce processus évolutionniste et des concepts; amateurisme, concept collectiviste, l'argent n'est pas essentiel et il se doit d'être partagé, professionnalisme, concept radicaliste (Halevy, 1904) avec une approche de conquête de richesse et sans partage.

Eléments de théorie

9 Pour percevoir les décisions stratégiques dans le paradigme rugbystique français d'aujourd'hui - l'acteur n'étant pas un individu mais des groupes humains -, dans ce contexte d'ébullition permanente d'ordre et de désordre instigateurs de création, nous sommes partis des acteurs pour découvrir le système (Crozier M. et Friedberg E., 1977), raisonnement heuristique dans la culture relationnelle du rugby français et ses multiples feed-back avec l'hémisphère Sud. De même pour la détection des intérêts, qui dépassent les préoccupations personnelles et immédiates de chacun d'eux, et la démonstration d'actions concrètes et des règles du jeu organisationnel qui s'appuient sur des sources et des zones d'incertitude - rugby professionnel mais pour qui et comment -.

10 Enfin considérant le changement comme phénomène adaptatif et inhérent à l'analyse systémique, le concept de jeu - l'instrument que les hommes ont élaboré pour régler leur coopération, la structure n'étant qu'un ensemble de jeux qui conduit au construit social - s'intégrait naturellement dans notre recherche sur les effets insufflés par l'émergence du professionnalisme, et qui sont l'épicentre de l'action collective. Des entretiens semi-directifs de dirigeants clés de la Fédération, de la CNRE, la Commission du Rugby de l'élite, de Comités Régionaux, représentants directs du rugby de masse, régional, des villages, et de joueurs et entraîneurs, associés à une analyse persistante de contenus intarissables tant dans la presse quotidienne que spécialisée, ont établi sans détour que chaque acteur représentant l'institution dont il dépend, est un éminent sujet transformateur. Les effets induits par les jeux de pouvoir qu'ils exercent (Mintzberg H., 1989), leur position sur l'échiquier organisationnel et leurs influences sur l'environnement ont conforté cette analyse systémique, mettant en relief la concurrence des différentes strates du système actuel braqué, directement ou indirectement, par la nouvelle exposition du rugby à la télévision, vers les satellites économiques représentés par les droits télés, les chaînes payantes, et dans leur sillage les sponsors. L'explication du phénomène social rugbystique, c'est encore relater la 
somme d'actions et ses résultats, et surtout en saisir le sens primaire et essentiel. La compréhension des actions des différents acteurs, c'est aussi admettre par le contenu d'informations authentiques, leurs ressources dans la situation actuelle et à venir, et de chacun d'entre eux dans le contexte social inhérent à leurs actions (Boudon R., 1989). Des processus récurrents qui dans ce système ouvert avec une entropie percutante, ne peuvent conduire qu'à un nouvel ordre (Morin E., Op. cit.) explicité par de nouvelles approches économiques, sociales et culturelles et reposant sur un postulat utilitariste de chaque acteur.

Les nouveaux liens, leurs stratégies, leurs conflitsLa métamorphose du paysage rugbystique français dans l'entropie

11 Face à la Fédération Française de Rugby, forte de 2000 clubs regroupant près de 300.000 licenciés, gestionnaire du rugby français orthodoxe et son Bureau Fédéral, Présidium suprême du Peuple d'Ovalie, font face la CNRE, Commission Nationale du Rugby d'Elite, créée en 1996, institution Palatine déclarée et avouée des partisans du professionnalisme et de l'élite et elle seule, l'Union des Clubs, représentative des 40 clubs des groupes $\mathrm{Al}$ et A2, largement associée aux desseins de la CNRE et depuis le 6 Mars 1998, créée dans l'urgence, l'Association des Clubs à vocation professionnelle. Vassales de la FFR, la CCDN, Commission des Clubs de Divisions Nationales, instaurée en 1996, prestataire des divisionnaires (groupe B, 2e et 3e division soit 338 clubs) et des "sans-grade», en quête de rugby sans tassette, et l'Amicale des Clubs du Rugby Français, créée en 1996, dotée de 170 clubs issus des divisions régionales, qui aspire à un rugby unitaire et se met au service de la CCDN. Aux côtés de ces institutions de collectifs s'ajoutent celles d'individuels, l'AJR, Association des Joueurs de Rugby, surtout de haut-niveau voire internationaux, créée en 1996 et dissoute en Février 1998 pour être rebaptisée Syndicat des joueurs, et l'UNER, l'Union Nationale des Entraîneurs de Rugby, initiée par la CNRE en 1996, qui regroupe les entraîneurs des groupes $\mathrm{Al}$ et A2. Tels sont les groupes d'acteurs officiels du système rugby français pour la saison 1997/1998 avec leurs jeux de pouvoir, leurs stratégies de négociation ou d'influence. Cette monographie pluridimensionnelle et synoptique est en inversion totale avec la monographie de Chapuis Jérôme (1988). 
Tableau $\mathrm{n}^{\circ} 1$. Source : Jacques Chapuis, "Mémoire entre amateurisme et professionnalisme ». Le rugby à XV au carrefour de son histoire? UFR STAPS Montpellier 1. 1998

\section{KLESUGY AUS QEBUT DES ANNEES T1980}

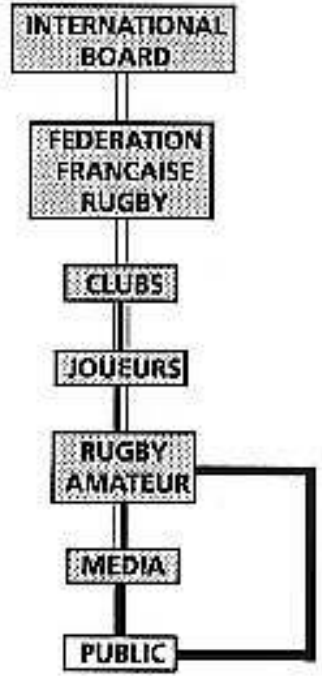

Lègende: tutelle $=$ responsabilitė $\equiv$ Productiondcrèation Information'critique

consommation

Tableau n ${ }^{\circ}$ 2. Source J.-B. Moles : Mémoire de Maîtrise 1997 UFR-STAPS Montpellier 1 , Ordre et désordre dans l'émergence du professionnalisme dans le rugby français.

\section{LE SYSTEME dU RUGBY FRANCAIS EN 1997}

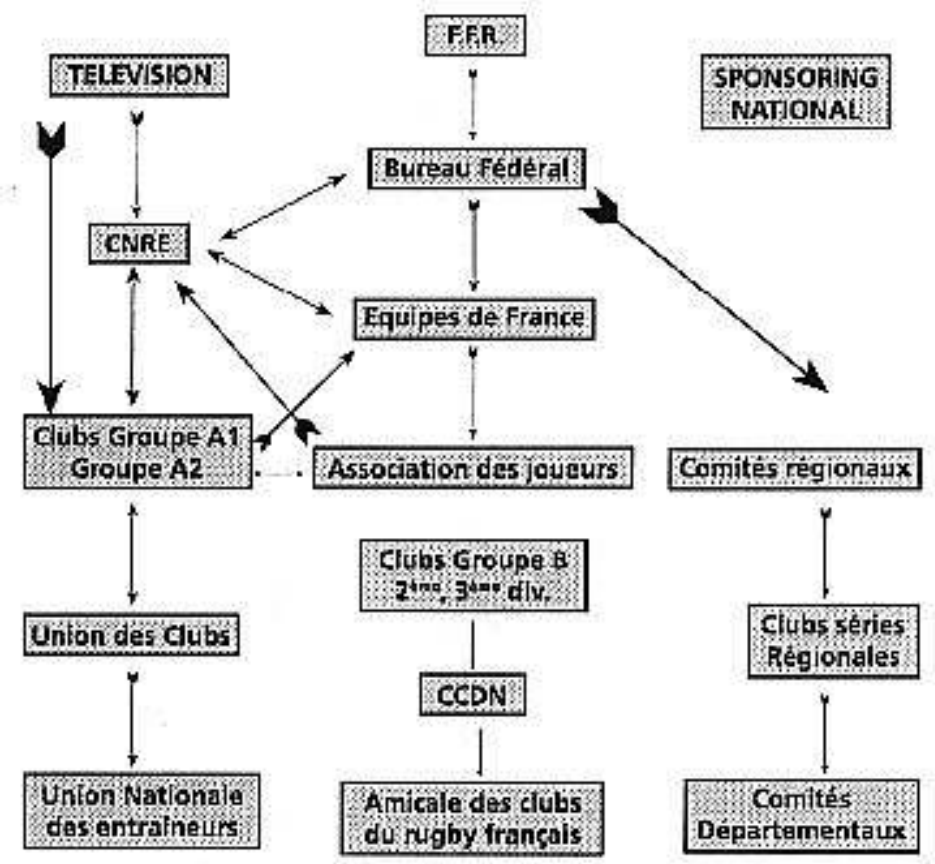

Dans cette monographie n'apparaît pas l'Association des Clubs à vocation professionnelle, créée seulement le 6 Mars 1998

12 Cette dernière monographie atteste d'une implosion du système sclérosé durant des lustres, et rend compte d'un système précurseur du professionnalisme. Notre démarche 
prospective dénote encore que l'interdépendance des acteurs, se traduit par le fait qu'aucune décision d'aucun d'entre eux ne peut être prise de façon unilatérale, sinon c'est le chaos, et parvenir à un compromis acceptable constitue le préalable indispensable à toute action. Ainsi en octobre 1997, aux souhaits de la CNRE d'installer dès la saison 1998/1999 une élite pure et dure de 16 clubs en poule unique, analogique au football, la FFR a répondu par une contre-proposition de 4 Provinces majeures (SudOuest, Midi-Pyrénées, Centre-Lyonnais et Ile-de-France) pour disputer en lieu et place des clubs, la Coupe d'Europe. A la culture des clubs, emblématique et prédominante dans l'Hexagone, la Fédération y opposait une culture de provinces, précipitée, anachronique et sans fondement historique. Cette tentative de déstabilisation de l'élite reçut un écho éminemment défavorable de celle-ci, qui la prit même pour une provocation publique. Les aveux de Bernard Lapasset, Président de la FFR reconnaissant que "ce n'était qu'une proposition ", mirent un terme à ce nouveau conflit mais ils ne firent que renforcer l'unicité élitaire, désormais orientée vers une stratégie d'indépendance totale vis-à-vis de la FFR, avec la création d'une Ligue externe. D'autant que celle-ci a été d'abord recommandé, puis finalement imposé en Mars 1998 (pour être officialisée avant l'été 1998), par le Ministère de la Jeunesse et des Sports, courroucée par la déambulation chronique des acteurs d'un système en état de chienlit permanente ; «Il ne s'agit pas d'une révolte, comme le pouvoir fédéral en a maté quelques-unes depuis des décennies, mais d'une révolution" (Carducci Jacques, L'Equipe, 15/10/1997). Patrick Sébastien, Président du C.A. Brive, renchérit ; "Que le Président arrête de nous cracher dessus, de nous donner tort dès qu'on fait une proposition. Qu'on cesse de nous opposer que du mépris. Soit le Président est prêt à écouter les clubs de l'élite et nous organisons la révolution avec lui, soit il faut qu'il s'en aille» (L'Equipe, 2 5/11/1997). Cette embardée témoigne de la désactivation latente du lien social entre les deux pôles prédominants de la restructuration du système rugbystique et de la situation sensible dans laquelle se retrouvent les petits clubs et l'institution qui les représente, la CCDN, qui clame; «Nous sommes favorables pour une élite qui soit la locomotive du rugby mais nous voulons vivre sans avoir à mendier 100 francs» (Raballo Serge, Président de la CCDN, Midi-Olympique, 10 Février 1997). Réponse des clubs de l'élite, "L'exposition du rugby se fait à la Télévision - le rugby est devenu en 1997 le second sport le plus exposé à la télévision en France, derrière le football (Source lettre du CSA, Décembre 1997) -, la télévision attend de nous $d u$ spectacle pour faire de l'audience. Pour faire du spectacle il faut de bons joueurs. Le football professionnel n'a pas tué le football amateur Le rugby d'Elite n'a évidemment pas les mêmes besoins que celui de la Masse » (voir tableau n ${ }^{\circ} 3$, sur l'évolution de l'exposition du rugby à Canal+). René Bouscatel, Président du Stade Toulousain, évalue ces besoins à «5MF budget nécessaire et suffisant pour une structure professionnelle» (Midi-Olympique, 15 Septembre 1997). Cette bipolarisation du rugby, avec d'un côté l'élite et le focus d'expansion braqué sur les droits télés, de l'autre la masse, attachée à un rugby unioniste et une politique de partage, met à nu les déterminismes marchands d'un côté, et de l'autre la volonté de ne pas se livrer corps et âme aux thuriféraires de l'affairisme, et le refus de délaisser une culture dont les racines sont ancrées dans la France profonde, les villes moyennes, bourgs et villages.

Tableau n³ (lettre du CSA, Décembre 1997)

Heures de diffusion de rugby par Canal+ 


\begin{tabular}{|l|l|l|}
\hline années & heures de diffusion & augmentation en \% \\
\hline \hline 1990 & $8 \mathrm{~h}$ & \\
\hline \hline 1993 & $22 \mathrm{~h}$ & $+280 \%$ \\
\hline \hline 1996 & $27 \mathrm{~h} 30$ & $+25 \%$ \\
\hline \hline 1997 & $48 \mathrm{~h}$ & $+77 \%$ \\
\hline
\end{tabular}

La bipolarité : le rugby des nantis, le rugby des humbles

La fracture sociale, la césure provoquée par l'émergence du professionnalisme, exprime des discordances qui font penser à la rupture du lien social, exacerbée par des valeurs marchandes et non-marchandes et qui rompt l'entraide dans l'ensemble des réseaux du système rugbystique français; "Le rugby professionnel est inévitable, avoue Serge Blanco, Président du Biarritz Olympique, c'est un mouvement irréversible. Mais qu'est ce qu'on met dedans? Qu'est ce qu'on fait? Il y a plein de questions et pas de réponses parce qu'on ne peut même pas les poser au Ministère puisque entre nous, clubs et Fédération, on n'est pas d'accord" (Equipe Magazine, $\left.n^{\circ} 814,8 / 11 / 97\right)$. Recréer des liens sociaux c'est apporter une solution à la désintégration du système actuel. Mais comment quand la distinction du rugby riche et rugby pauvre n'a jamais été aussi éloquente. Le budget moyen des clubs de l'élite pour la saison $97 / 98$ va de 23 MF à 9 MF pour les plus modestes. Le budget moyen des clubs du groupe B est de $1 \mathrm{MF}$, de 60.000 francs pour les clubs de séries régionales (près de 400 fois moins que le budget de Brive). Des valeurs morales, le rugby est passé à des valeurs économiques intangibles. Certes les passions humaines restent fertiles, mais les moyens pour l'action sont diamétralement opposés ne laissant aux modes de relation qu'une filiation abusive entre les clubs riches et les clubs pauvres. Les liens d'interconnaissances (Tonnies F., 1887) qui se développent dans ce nouvel espace social ne sont plus fondés sur l'acceptation de règles de conduite similaires (professionnalisme et amateurisme). D'autant que l'approche décisionnelle qui présuppose que les membres de l'organisation situés dans une position inférieure laissent de côté leur autonomie, est obsolète (Simon H. A., 1957). La virtualité d'un dénominateur commun autorise cependant le Président de la FFR à déclarer "il faut aussi définir un projet sportif pour les petits clubs qui vivent au sein d'une communauté rurale très forte ». Paroles pieuses mais pour quel écho? "Dans un système de dictature de marché, les clubs les plus riches vont devenir de plus en plus riches et les plus pauvres seront laminés " (Bourg J.-F., L'Equipe Magazine, n782, Mars 1997). Cette extinction des modestes, qui aux droits télés opposent un droit de survie, c'est aussi l'extinction de la culture de l'apprentissage dans les écoles de rugby dès le plus jeune âge, du courage, de la vaillance, de l'altruisme, de la solidarité qui forgent les caractères et laissent une empreinte indélébile chez ceux qui, plus tard, "mouilleront le maillot ", " sueront le sang " et deviendront des bâtisseurs de légende comme Michel Crauste, le Mongol Lourdais, Amédée Domenech, le Duc Briviste, Alfred Roques, le Pépé du Quercy, Oualtère, Spanghero le Narbonnais, André Hérrero, le corsaire toulonnais, JeanPierre Rives, le Blond. Ces figures mythiques nourrissent sans faillir par leur mémoire le patrimoine d'Ovalie et perpétuent l'affectif, sève de l'idéal collectif quinziste. Mais aujourd'hui le mythe n'est qu'actualité, la légende... magazine, les héros... athlètes, car 
les règles en perpétuelle mouvance ont doublé le temps de jeu effectif qui dépasse régulièrement les 30 minutes. L'élite n'est plus la panacée d'un amateurisme «marron». Seuls les professionnels ou semi-professionnels peuvent y prétendre. Le charisme n'est plus essentiel, le rendement seulement. L'avenir est subodoré en francs, les «capes» valorisent davantage les contrats que la notoriété. La bravoure est quantifiée, la vaillance n'est que subséquente à la rigueur et à la méthode. Tout est fait pour tendre vers un produit spectaculaire. D'autant mieux que les télévisions sont demandeuses, $144 \mathrm{~h} 23^{\prime}$ de diffusion en 97 contre $402 \mathrm{~h} 17^{\prime}$ au football, ce qui représente un ratio en tenant compte du nombre de licenciés en football 1.962 .000 contre 261.700 pour le rugby, très avantageux pour celui-ci, 7,49 (licenciés), 2,79 (heures de diffusion télé) (Equipe Magazine, N783, Avril 1997).

Les téléspectateurs en hausse, les spectateurs en baisse

Le commensalisme public/rugby avec l'émergence du professionnalisme et l'implication grandissante de la télévision, a subi depuis deux ans un véritable coup d'arrêt car les supporters appréhendent avec peine cette métamorphose économique et culturelle du jeu, au point que les spectateurs payants s'effacent dangereusement. En un an le Groupe A a perdu près de 30.000 spectateurs soit une baisse de 6.4\%. Baisse encore plus conséquente en Groupe A2 -7,8\%.

Tableau n4. Sources Midi-Olympique 1997

\begin{tabular}{|l|l|l|l|}
\hline Groupes A1 & $95 / 96$ & $96 / 97$ & $\%$ \\
\hline \hline Entrées payantes & 418.292 & 391.296 & $-6,4 \%$ \\
\hline \hline Recettes & $26,690 \mathrm{MF}$ & $25,732 \mathrm{MF}$ & $-3,6 \%$ \\
\hline
\end{tabular}

15 Un public qui fût toujours à l'antinomie de celui du football, ignora le hooliganisme et les incantations grossières. La complexité des règles en rugby tend il est vrai à une plus grande réflexion sur le jeu et sur ses concepts, les décisions arbitrales sont disséquées, commentées, rarement conspuées. Ce public friand de luttes épiques et de derbys enflammés découvre un nouveau rugby, un nouveau jeu (l'ascenseur en touche de tout temps prohibé, est depuis 1995 une phase de jeu essentielle pour l'appropriation de la balle, la pénalité, outil de réalisation prédominant depuis des décennies, est devenue une structure d'approche et un relais pour la continuation du jeu. Le lien culturel entre le public et les pratiquants de ce rugby néo-contemporain se distend, les recettes en font foi, même si des rencontres entre les grosses écuries de l'élite attirent encore foule. Heureusement d'ailleurs. De même l'impact des grands événements, le Tournoi des V Nations, (France/Angleterre au Stade de France le 7 Février 1998 a rassemblé 76.000 spectateurs), la finale de la Coupe d'Europe "Heineken Cup " (Brive/ Bath à Bordeaux le 31 Janvier 1998 s'est jouée à guichets fermés), reste intact. Mais la nouvelle puissance télévisuelle, avec l'exposition inattendue du rugby, la réalisation de plus en plus appliquée, qui restitue à ce jeu une intense émotion et une amplitude de jeu de mouvement jamais atteinte, devraient aider à réconcilier les partisans du rugby orthodoxe avec ce rugby néophile et les ramener vers le championnat, si celui-ci retrouve cependant un calendrier et une formule cohérentes (trois formules ont été successivement décidées au cours de cette année pour la future saison!). 
Il n'y a plus d'amour entre joueurs

16 Les joueurs de rugby, professionnels ou semi-professionnels, eux sont déjà dans une phase de mutation complexe. L'entraînement, de trois fois hebdomadaire, est devenu aujourd'hui quotidien voire biquotidien. Philippe Saint-André, capitaine du XV de France en 1997, avouait pour le seul mois d'octobre 1997, 58 entraînements, auxquels il faut ajouter les matches! Le suivi médical devient de plus en plus performant, avec ostéopathes et diététiciens. Les temps de récupération, honnis dans un passé récent, sont aujourd'hui sacrés. La prévalence de l'esprit collectif, dans une société de plus en plus individualiste, reste prégnant car en rugby le combat n'est pas celui d'un homme mais d'un groupe. Et c'est dans ce collectif que se nouent solidarité et altruisme, reconnaissance et générosité, fierté et abnégation et même amour, mais selon Gilles Bourguignon, 2e ligne Narbonnais, international «Il n'y a plus cet amour entre les joueurs de l'élite. Pas plus entre les joueurs et les supporters. Les joueurs pensent trop à l'argent et pas assez aux relations humaines et sociales». Des propos corroborés par Pierre Berbizier, excapitaine et entraîneur du XV de France, "Le rugby est un sport collectif de combat qui réclame beaucoup de solidarité. Or rien que sur les contrats de la Charte en équipe de France, on sort 10 joueurs qui touchent 400.000 Frs et les autres rien. C'est un consensus de complaisance " (L'Equipe, 25/1 Y 1997). Serge Blanco, historique capitaine du XV de France, constate aussi cette érosion sociale et de vie de groupe mais pour lui "C'est le contexte qui est différent, c'est tout. C'est vrai qu'autrefois les joueurs restaient beaucoup entre eux et les femmes attendaient à la maison. C'était des moments privilégiés. Aujourd'hui la vie de groupe c'est une fois de temps en temps, mais c'est la vie qui change, ce n'est pas le rugby qui change les mentalités 》 (Equipe Magazine, $\mathrm{N}^{\circ} 814,8$ Novembre 1997).

Autre variable d'effet de l'émergence du professionnalisme, le rugby philosophie de vie et de culture, bousculé par les exigences du réalisme économique, a du mal à perdurer dans le monde universitaire (sur 235.000 licenciés en 1997, 135.000 sont scolaires ou universitaires soit 57,45\%). L'incompatibilité des études et de l'entraînement de hautniveau est aujourd'hui suffisamment éloquente pour que Fred Artigot, entraîneur des équipes universitaires de Paul Sabatier de Toulouse, plusieurs fois championnes de France, s'en émeuve sans complaisance - "On s'achemine doucement vers l'impossibilité de faire des études et du rugby de haut-niveau, même si quelques clubs se penchent encore sur le devenir social». Et Louis Gagniéres, le Président du RC. Nîmes, qui a toujours privilégié la politique de formation à celle d'un recrutement argenté d'avertir "On prépare de futurs SDF et une cohorte de chômeurs en crampons ". Les écoles de rugby, même les plus illustres, faute de bénévoles s'étiolent et les clubs de villages, sponsors et supporters momifiés par les diffusions télévisées crient à la famine ; «Le rugby est prisonnier de l'argent, de la télévision (Théorème de Murdoch). Lorsque j'entends les sommes qui sont redistribuées, lorsque je vois la galère financière des petits clubs, je ne peux que constater l'écart qui est en train de se creuser Nous sommes au bout du compte aux prémices d'une inévitable mutation» (Eric de Garets, Président de Saint-André de Cubzac). Ces écoles, ces petits clubs sont pourtant l'essence de la formation, le vivier des grand clubs, la base du rugby unitaire, et bien sûr ils crient halte à cette philosophie anachorétique et «à ces intrus qui veulent goûter notre pain sans connaître l'origine de la farine " (Pierre Albaladéjo, 1997). Ils appellent au partage, à la solidarité et à la sauvegarde de la promotion sociale pour que le rugby de mercenaires et l'appel des métropoles riches, coeur génétique du rugby élitiste, ne laissent pas exsangue d'autres villes moyennes comme le sont déjà celles, légendaires dans l'histoire du rugby orthodoxe, de Lourdes, Bagnères de Bigorre, Le Creusot, Bourg- 
en-Bresse, Tarbes, Carmaux, Lavelanet, La Voulte, Prades, Thuir, Carcassonne, Quillan et tant d'autres.

Une césure irréfutable

18 La césure sociale devient irréfutable car les deux rugbys, élitaire et unitaire n'ont plus rien de commun, ne défendent plus les mêmes valeurs, n'ont plus les mêmes intérêts. Mais tout n'est pas aussi simpliste. Pour les partisans de l'élite, l'argent est le nerf de la guerre, or, nous l'avons précédemment relaté, les ressources directes, les entrées payantes, baissent. Les ressources des sponsors sont aussi en constante régression. Dès lors comment parvenir au professionnalisme? Seul et ultime palliatif, les droits télévisés. Mais ils frôlent aujourd'hui, en comparaison avec le football, le ridicule : 28 MF minimum pour chaque club de football de 1 ère division pour le championnat de France 97/98, 600.000 Frs pour le Stade Toulousain, champion de France de rugby en 1997 ! Près de 50 fois moins! Pour la saison 1996/1997, la somme versée à l'ensemble des clubs de l'élite par la CNRE, grâce à la Caisse de Blocage des droits télés du championnat, de la Coupe de France et de la Coupe d'Europe, s'est élevée à 39 MF soit à peine plus que pour un seul club de football de 1 ère division (droits de championnat et de Coupe de la Ligue). Cette caisse de blocage a permis à Bourgoin de percevoir $2 \mathrm{MF}$ et à un club du groupe A2 comme Istres, 603.000 frs. Mais Bourgoin guette du côté anglais, où les clubs participant au championnat de l'élite ont reçu comme Northampton, $7 \mathrm{MF}$ de droits télés et marketing et 2,3 MF de la Fédération. Près de 10MF! Aussi tant à Brive, Toulouse, le Stade Français, Bourgoin, antres du préprofessionnalisme, le temps n'est plus à se pencher sur le sort des humbles. A chacun ses problèmes et ses soucis. Le rugby de l'élite a besoin de la télévision et ses droits ( 8 MF pour Canal+ et 51 MF pour France Télévision en 98) pour parvenir au professionnalisme. Le rugby unitaire a besoin du soutien de la Fédération pour s'affirmer. Et la FFR dont le budget est de $350 \mathrm{MF}$, l'équivalent du PSG football, lorgne sur ces mêmes droits télés (qu'elle perçoit directement) pour asseoir sa tutelle sur ce rugby unitaire et réprimer les ardeurs élitaires. Un irréversible processus récursif qui touche le degré d'autonomie des deux pôles, élitiste et unitaire, et qui est le déclencheur de l'instabilité actuelle et prochaine, quand les droits télés seront à discuter (pour Canal+ en 98 et France Télévision en 99). L'ensemble du système n'a donc aujourd'hui plus aucune cohérence et leurs 'interrelations semblent totalement irrationnelles si on édulcore les enjeux, mais sont finalement rationnelles si l'on en connaît l'importance. Ce que nous n'ignorons point. Cette contradiction offre à l'environnement un rôle inattendu de co-organisateur, puisque le mode de compétition, le calendrier, le «merchandising » et les dotations des équipementiers dépendent de la télévision. En 1990 Adidas versait 150.000 Frs de dotation en équipements aux clubs de 1 ère division. En 1997, l'exposition télévisée aidant, la dotation grimpe à 500.000 Frs pour les grands clubs de l'élite.

Le système du rugby français défait manifestement ses liens sociaux au profit de liens économiques. L'enjeu social ne pèse rien face à l'enjeu économique. Et Si les liens sociaux se renouent cela ne se fera que par le bon vouloir des liens économiques. C'est cela la grande leçon de la tentative du rugby français élitiste de se positionner dans le camp des révisionnistes. La CNRE, dans cette perspective aléatoire mais audacieuse, a déjà précisé à Madame Buffet, Ministre de la Jeunesse et des Sports, que dès la création de la Ligue (isomorphe de la Ligue de football), elle disparaîtra pour laisser à celle-ci le soin de gérer l'avenir du rugby professionnel hexagonal, en indépendance totale de la Fédération, ne laissant que comme seules compétences à la FFR celles intégrant les équipes de France et la section amateur (une hypothèse rendue d'ailleurs obligatoire, 
car la loi du 13 Juillet 1992 précise que les clubs enregistrant des recettes horssubvention de plus de 2,5MF et distribuant des rémunérations pour le même montant doivent adopter le statut de SAOS (Société Anonyme à Objet Sportif), ou SAEMS (Société Anonyme d'Economie Mixte Sportive) - à ce jour tous les clubs de l'élite sont donc dans l'illégalité -. La Fédération espère que cette structure demeurera cependant à vocation interne misant sur l'instabilité des stratégies qui se confinent dans cet enchevêtrement extrême. Mais la cogestion du système rugbystique français par deux pôles antinomiques est associée depuis un an, à un tel faisceau d'incohérences, tant les liens sociaux sont banalisés par le souci de rupture et les liens affectifs galvaudés par l'économie de marché, "l'argent à tout foutu en l'air » (Albert Ferrasse, ex-président de la FFR), que cette hypothèse parait bien utopique tellement les discours de chacune des parties, élitiste et unitaire, sont marqués. "Je ne veux pas être le Président d'une Fédération de chômeurs» (Bernard Lapasset, Président de la FFR), "Il n'est pas question que nous devenions les SDF du rugby» (Serge Raballo, Président de la CCDN), "Il faut faire avec l'argent qu'il y a et qu'on n'attende pas de miracle " (Séraphin Berthier, Président de la CNRE). Entre le refus logique du " rugby misère », du "rugby chômage et de devenir SDF " (discours très prégnant dans le rugby unitaire), et l'objectif ambitieux d'un rugby argenté mais qui cherche toujours la richesse (les joueurs du Montpellier RC. se sont mis en grève en Novembre 1997 - une première dans le champ social rugbystique français - leurs salaires n'étant plus versés depuis 2 mois, la moitié de l'effectif a quitté le club en cours de saison), le système du rugby français n'a pas fini de nous interpeller sur sa complexité même s'il est envisageable qu'en 1999 il évolue dans un paradigme de simplicité avec des relations roboratives, formelles, distinctes et logiques entre les deux notions maîtresses (rugby élite/rugby de masse, Ligue/Fédération).

Les passions, laudatives, les valeurs des contributions à la prospérité morale et sociale du rugby, et la reconnaissance d'une filiation entre la base et la hiérarchie (Juffé M., 1995), viscérales dans le rugby orthodoxe, affects tangibles à cette césure, établissent une conception matérialiste de faits socioculturels rugbystiques, et nous amènent à privilégier les activités économiques pour déterminer une planification stratégique du nouveau système. Ce premier indicateur de finalisation de césure sociale est la concrétisation d'une acculturation sur la culture treiziste de l'hémisphère Sud dont la contemporanéité est dimensionnelle à celle du système quinziste des Antipodes (évacuation expresse de l'amateurisme, libéralisme dipolaire - nations riches et nations démunies -, assujettissement aux lois cathodiques, concentration élitaire). Si césure effectivement il y a entre le rugby élitiste et unitaire, entre rugby de terroir et rugby spectacle, il va falloir repenser l'espace social dans lequel tous les acteurs du rugby hexagonal vont vivre. Laissant, pour exemple, le rugby élitiste évoluer sur un mode américain avec un championnat de franchisés. La culture rugbystique française est-elle prête à admettre pareille extrapolation même si le Stade Français avec NRJ et Castres avec les laboratoires Fabre, sous connotation corporative ont bien intégré ce concept marketing? De même en se liant aux droits télés comme le football et la Ligue avec Canal Plus, le rugby est-il prêt à la rémission de ces ultimes idéaux, la convivialité, la déstarisation, la 3e mi-temps, un public paternaliste et éclairé?

La gestion du rugby orientée par des structures capitalistes pour optimiser ses ressources et profits, fait d'un joueur un acteur, hautement qualifié, d'un supporter un client, d'une équipe un support commercial car l'aboutissement est de vendre du spectacle; «Le rugby est devenu un business quand vous avez 75.000 spectateurs dans le stade, des millions de gens qui sont devant leur poste télé et qu'il y a autant d'argent enjeu, précise 
Clive Woodward l'entraineur du XV d'Angleterre " (L'Equipe, 6/02/1998). Gagner n'est plus suffisant, il faut plaire, et pour l'avoir omis en septembre 1997, les clubs qualifiés en Coupe d'Europe ont vu avec effroi France Télévision, supprimer sans ambages les diffusions de leurs rencontres le samedi en access time.

En 1823 Webb Ellis, ballon de football sous le bras, termina sa folle course par un plongeon historique derrière les buts, tel pour s'extirper du système footballistique. Le Peuple d'Ovalie, nostalgique et à la mémoire féconde, nous rappelle que le rugby orthodoxe est né de cet artefact. Le professionnalisme dans le rugby n'est rien d'autre qu'un nouvel artefact. S'il connaît la même épopée que le précédent nul n'aura à se plaindre de cette nouvelle exclusion. Mais de cette désintégration du système du rugby unitaire due à la dissolution des liens de dépendances mutuels, qui auraient pu permettre le maintien de l'ordre socio-rugbystique, peuvent et doivent surgir de nouveaux liens entre une communauté avant-gardiste et résolument mercantile, et l'autre traditionnelle et garante d'un idéal d'amateurisme, passerelle authentique entre l'élite et la masse, la future Ligue et la Fédération. Pour que le rugby cesse avec l'insécurité sociale et renoue avec l'utilité sociale.

\section{BIBLIOGRAPHIE}

Boudon R. (1989) Effet pervers et ordre social. Paris. PUF.

Bourdieu P (1979) La distinction. Critique sociale du jugement. Paris. Ed. de Minuit.

Chapuis J. (1988) Mémoire entre amateurisme et professionnalisme. Le Rugby à XV au carrefour de son histoire. Mémoire de maîtrise. UFR STAPS Montpellier inédit.

Crozier M. et Friedberg E. (1977) L'acteur et le système. Paris. Seuil.

Durkheim E. (1968) Education et sociologie. Paris. PUF.

Escot R. (1996) Rugby Pro. Histoires secrètes. Paris. Solar.

Juffé Michel (1995) Les fondements du lien social. Paris. PUF.

Hallevy. (1904) Formation du Radicalisme philosophique.

Mintzberg H. (1986) Le pouvoir dans les organisations. Les Editions d'Organisation.

Morin E. (1986) La Méthode. Paris. Seuil.

Morin E. (1990) Introduction à la pensée complexe. Paris. ESF.

Pareto V (1916-1968) Traité de sociologie générale. Genève. Droz.

Parsons T. (1951) The social System. NY. Free Press.

Rugbyrama (1996-1997) Toulouse. Ed. La Dépêche du Midi.

Rey J.-P. (1997) Qu'ont-ils fait de notre rugby. Paris. Solar.

Simon H.A. (1957) in La Gestion des Organisations sous la direction de Bédard M.G. et Miller R. Chenelière/Mc Graw-Hill. 
Tônnies F (1977) Communauté et société. Paris. Retz. CEPL.

\section{RÉSUMÉS}

En août 1995, l'International Board, l'institution suprême du rugby mondial, sous la pression de l'hémisphère Sud, gomme de ses statuts toute référence alors obligatoire à l'amateurisme. Le rugby devient un sport professionnel. Cette mutation provoque, surtout dans l'hémisphère Nord et notamment en France, la désintégration du rugby unitaire en deux camps, l'élite et la masse, et la dissolution de liens de dépendances mutuels. Cette situation peut-elle perdurer?

In august 1995, The International Board, the head institution of World Rugby, under the pressure of South Hemisphere, withdrew its statutes all references, at that time compulsory for the amateurism. Rugby becomes a professional sport. This change causes, especially in the North hemisphere and particularly in France, the breaking up of the united rugby in two parts, the elite and the masse, and the dissolution of mutual dependence links. Could this situation go on?

\section{INDEX}

Keywords : culture, élite, masse, disorder, professionnalism

Mots-clés : désordre, professionnalisme

\section{AUTEUR}

\section{JEAN-BERNARD MARIE MOLES}

Faculté des Sciences du Sport et de l'Education Physique. Université Montpellier 1. Equipe «Corps et Culture » 